hep-th/9604198

RU-96-24

\title{
Gauge Fields and D-branes
}

\author{
Michael R. Douglas \\ Department of Physics and Astronomy \\ Rutgers University \\ Piscataway, NJ 08855-0849 \\ mrd@physics . rutgers . edu
}

\begin{abstract}
We prove that self-dual gauge fields in type I superstring theory are equivalent to configurations of Dirichlet 5-branes, by showing that the world-sheet theory of a Dirichlet 1-brane moving in a background of 5-branes includes an "ADHM sigma model." This provides an explicit construction of the equivalent self-dual gauge field. We also discuss type II.
\end{abstract}

April 1996 


\section{Introduction}

Recent work has demonstrated the power of the Dirichlet brane [2, 3] in constructing highly non-trivial solutions of superstring theory [4].

Although at first glance these solutions look very different from those in which a conventional metric and gauge fields satisfy conventional equations of motion (with stringy corrections), it is known that in many cases they are to be regarded as extreme limits of field theory solutions, singular in field theory but non-singular in string theory. This stringy resolution of the singularity can already be seen in the low energy limit, as due to additional massless degrees of freedom in the effective field theory.

The example currently best understood is the Yang-Mills instanton. In type I superstring theory this becomes a 5-brane with the appropriate charge and action to be a Dirichlet brane. Witten argued that the D5-brane world-volume is identical to the zero scale size limit of the instanton [5], by showing that the space of vacua of a system of parallel D5-branes is isomorphic to the moduli space of instantons on $\mathbb{R}^{4}$. Indeed, the physical definition of these vacua (solutions of D-flatness conditions of a supersymmetric gauge theory) is identical to the ADHM construction [6], which produces instanton moduli space as a hyperkähler quotient.

The ADHM construction also provides an explicit construction of the actual gauge potential for these instantons. To reproduce this physically, we should insert a very heavy charged probe, point-like in the four dimensions transverse to the 5-branes whose position is well defined. Its motion will then depend locally on the vector potential defined at a point.

The natural probe is another D-brane. A D1-brane extending parallel to the 5-branes will be pointlike in the four dimensions containing the instanton, and its world-sheet action will be a sigma model describing propagation of a heterotic string soliton in this gauge field. It has $(0,4)$ supersymmetry and its field content was described in [7]. The 5 and 9-brane fields will play the role of couplings. Since these are ADHM data, we might expect the world-sheet theory to be equivalent to Witten's ADHM sigma model [8]. We now show that this is true.

\section{Type I}

Consider a 1-brane moving in the presence of $k 5$-branes and $N 9$-branes in type I theory. We will conform to the notations of [8,9,10] where possible.*

\footnotetext{
* Notations we changed: $X$ in [8] is now $b ; Y$ in [7] is now $\phi$.
} 
We take 1-branes in the 01-plane, and 5-branes in 012345. The Lorentz symmetry becomes $S O(1,1) \times S O(4)_{I} \times S O(4)_{E}$. We index doublets of the two $S U(2)$ 's in $S O(4)_{E}$ as $A$ and $Y$, and doublets of the two $S U(2)^{\prime}$ 's in $S O(4)_{I}$ as $A^{\prime}$ and $\tilde{A}^{\prime}$. All of these indices will be raised and lowered as $v_{A}=\epsilon_{A B} v^{B}$. The $(0,4)$ supersymmetries are parameterized by $\eta_{+}^{A A^{\prime}}$. We index 5 -branes with $m$ and 9 -branes with $M$. The indices $m$ will be raised and lowered with the $S p(2 k)$ invariant antisymmetric tensor $\epsilon^{m n}$. For consistency with [8], let the index $a$ be the union of choices from $M$ and $Y \times m$, and let $Y^{\prime}$ be an alternate name for $m$.

The 5-brane fields include $S p(2 k)$ gauge theory, hypermultiplets in the antisymmetric tensor representation, and "half-hypermultiplets" in the $(2 k, N)$ of $S p(2 k) \times S O(N)$. Their scalar components are $X^{A Y}=\epsilon^{A B} \epsilon^{Y Z} X_{B Z}^{*}$ and $h_{M}^{A m}=i \epsilon^{m n} \epsilon_{A B}\left(h^{*}\right)_{B n}^{M}$. These will become couplings in the 1-brane world-sheet theory.

The $(0,4)$ multiplets come in two varieties, 'standard' with $\delta b^{A i}=\eta_{A^{\prime}}^{A} \psi^{A^{\prime} i}$, and 'twisted' with $\delta \phi^{A^{\prime} j}=\eta_{A}^{A^{\prime}} \chi^{A j}$.

The world-sheet fields are then

boson fermion sector

$$
\begin{array}{cccc}
b^{A Y} & \psi_{-}^{A^{\prime} Y} & D D & \text { positions transverse to } 5 \text {-brane } \\
b^{A^{\prime} \tilde{A}^{\prime}} & \psi_{-}^{A} \tilde{A}^{\prime} & D D & \text { positions within } 5 \text {-brane } \\
\phi^{A^{\prime} m} & \chi_{\overline{-}}^{A m} & D N 5 & 1-5 \text { strings } \\
& \chi_{+}^{Y} m & D N 5 & \text { GSO correlates } S O(1,1) \text { and } S O(4)_{E} \text { chirality } \\
& \lambda_{+}^{M} & D N 9 & \text { GSO selects } S O(1,1) \text { chirality } \\
& \lambda_{+}^{a} & & \text { An alternate notation for the set }\left(\chi_{+}^{Y m}, \lambda_{+}^{M}\right) .
\end{array}
$$

Here $b^{A Y}=\epsilon^{A B} \epsilon^{Y Z} \bar{b}_{B Z}$, and $\phi^{A^{\prime} m}=\epsilon^{A^{\prime} B^{\prime}} \epsilon^{m n} \bar{\phi}^{B^{\prime} n}$. The $\lambda$ 's are real.

It is useful to first consider subsectors involving a subset of the branes. We will use the fact that upon combining subsectors, the action is the sum of the action for each subsector, plus possible new couplings involving more than one subsector. The 19 subsector of $b, \psi$ and $\lambda$ preserves $(0,8)$ supersymmetry and is just the heterotic soliton theory of [1] with a free action.

The 15 subsector can be derived by dimensional reduction of a $d=6, \mathcal{N}=159$ theory in the 016789 plane. Due to the $\Omega$ projection this is an "SO(1) gauge theory," but nevertheless the couplings to $b^{A Y}$ are determined. They give the DN modes $\phi$ and $\chi$ a mass proportional to the distance between the 1 and 5-branes. Translation symmetry in the 6789 plane allows only the combination $\left(X_{m \bar{n}}^{A Y}-b^{A Y} \delta_{m \bar{n}}\right)$ to appear:

$$
\begin{aligned}
\mathcal{L}=\mathcal{L}_{\text {free }}+ & \phi_{A^{\prime} m} \phi^{A^{\prime} n}\left(X_{m n}^{A Y}-b^{A Y} \delta_{m n}\right)^{2}+ \\
& \chi_{-}^{A m} \chi_{+}^{Y n}\left(X_{m n}^{A Y}-b^{A Y} \delta_{m n}\right)+\psi_{-}^{A^{\prime} Y} \chi_{Y+}^{m} \phi_{A^{\prime} m}+\text { c.c. }
\end{aligned}
$$


(and couplings to the fermionic partners of $X$ ). The last of these is required by $(0,4)$ supersymmetry.

The 159 couplings respect only $(0,4)$ supersymmetry. These can be computed by world-sheet techniques; for example

$$
\mathcal{L}=\lambda_{+}^{M} \chi_{m-}^{A} h_{A M}^{m}
$$

comes from a correlator of three twist fields, which is evidently non-zero.

By now imposing $(0,4)$ supersymmetry, we have enough information to get the complete Lagrangian. In [8] the general $(0,4)$ Lagrangian with this field content was worked out, and is given in equation (2.21):

$$
\begin{aligned}
L=L_{\text {free }} & -\frac{i}{4} \int d^{2} \sigma \lambda_{+}^{a}\left(\epsilon^{B D} \frac{\partial C_{B B^{\prime}}^{a}}{\partial b^{D Y}} \psi_{-}^{B^{\prime} Y}+\epsilon^{B^{\prime} D^{\prime}} \frac{\partial C_{B B^{\prime}}^{a}}{\partial \phi^{D^{\prime} Y^{\prime}}} \chi_{-}^{B Y^{\prime}}\right) \\
& +\frac{1}{8} \int d^{2} \sigma \epsilon^{A B} \epsilon^{A^{\prime} B^{\prime}} C_{A A^{\prime}}^{a} C_{B B^{\prime}}^{a} .
\end{aligned}
$$

The functions $C$ determine the supersymmetry transformations of the left moving fermions

$$
\delta \lambda_{+}^{a}=\eta_{+}^{A A^{\prime}} C_{A A^{\prime}}^{a}
$$

and are determined in terms of four constant matrices $M, N, D$ and $E$ :

$$
C_{A A^{\prime}}^{a}=M_{A A^{\prime}}^{a}+b_{A Y} N_{A^{\prime}}^{a Y}+\phi_{A^{\prime}}^{Y^{\prime}} D_{A Y^{\prime}}^{a}+\phi_{A^{\prime}}^{Y^{\prime}} b_{A Y} E_{Y Y^{\prime}}^{a}
$$

The condition for $(0,4)$ supersymmetry is then

$$
0=\sum_{a} C_{A A^{\prime}}^{a} C_{B B^{\prime}}^{a}+C_{B A^{\prime}}^{a} C_{A B^{\prime}}^{a}
$$

Comparing with (2.1) determines

$$
C_{A A^{\prime}}^{Y m}=\phi_{A^{\prime} n}\left(X_{m n}^{A Y}-b^{A Y} \delta_{m n}\right)
$$

The coupling (2.2) will be reproduced by

$$
C_{A A^{\prime}}^{M}=h_{A m}^{M} \phi_{A^{\prime} m}
$$

which also implies the existence of an $h^{2} \phi^{2}$ term in the potential.

This completes the derivation of the Lagrangian, and indeed the couplings of the sigma model are given by ADHM data. Thus the 1-brane world-volume theory is essentially Witten's ADHM sigma model (with the additional free fields $b^{A^{\prime} \tilde{A}^{\prime}}$ and $\psi_{-}^{A \tilde{A}^{\prime}}$ ). The condition for $(0,4)$ world-sheet supersymmetry (2.6) is

$$
0=h_{m}^{M(A} h_{M n}^{B)}+\epsilon^{p q} \epsilon_{Y Z} X_{m p}^{(A Y} X_{n q}^{B) Z}
$$

which is just the D-flatness condition for the 5-brane theory. As is often the case, the conditions for extended supersymmetry in the sigma model are the equations of motion in the target space. 


\subsection{The ADHM connection}

Integrating out massive degrees of freedom on the 1-brane will produce a conventional gauged sigma model. This was done explicitly in [8], to produce the self-dual gauge connection corresponding to the ADHM data $X$ and $h$.

For generic ADHM data $X \neq 0$, the potential $V=\sum C^{2} \sim \phi^{2}\left(X^{2}+b^{2}\right)$ gives mass to $\phi$, leaving the fields $b$ to describe the location of the 1-brane in four dimensions. The Yukawa couplings of (2.3), (2.7) and (2.8) become, taking $\phi=0$, and suppressing the $m n M N$ indices,

$$
\left(\begin{array}{ll}
\chi_{1} & \chi_{2}
\end{array}\right)_{-} \cdot\left(\begin{array}{ccc}
X^{11}-b^{11} & X^{12}-b^{12} & h_{M}^{1 m} \\
X^{21}-b^{21} & X^{22}-b^{22} & \left(h^{2}{ }^{t r}\right)_{m}^{M}
\end{array}\right)\left(\begin{array}{c}
\chi^{1} \\
\chi^{2} \\
\lambda
\end{array}\right)_{+}
$$

These couplings will pair $\chi_{-}$with $4 k$ fermions from the set $\lambda_{+}^{a}$, leaving an $N$-dimensional subspace of massless modes varying with $b$. This bundle of massless modes is the resulting gauge bundle, and projecting the original flat connection onto this subbundle will produce the ADHM connection.

Referring to the operator in (2.10) as $\mathcal{D}^{+}$, the subbundle is $E \equiv$ ker $\mathcal{D}^{+}$. Given an explicit orthonormal basis $v_{i}^{a}(b) \in E$, the connection can be written by changing variables to massless fermions $\lambda_{i+}$ :

$$
\lambda_{+}^{a}=\sum_{i=1}^{N} v_{i}^{a}(b) \lambda_{i+} .
$$

Inserting this in the kinetic term $\lambda_{+}^{a} \partial_{-} \lambda_{+}^{a}$ produces the Lagrangian

$$
\begin{aligned}
\mathcal{L} & =\lambda_{i+}\left(v_{i}^{a}(b)\right)^{-1} \partial_{-} v_{j}^{a}(b) \lambda_{j+} \\
& =\lambda_{i+}\left(\delta^{i j} \partial_{-}+A_{A Y}^{i j}\left(b^{A Y}\right) \partial_{-} b^{A Y}\right) \lambda_{j+}
\end{aligned}
$$

with $A_{A Y}^{i j}(b)=\left(v_{i}^{a}\right)^{-1} \partial v_{j}^{a} / \partial b^{A Y}$.

A last point to mention is that there can also be a direct coupling to the gauge field on the 9-branes,

$$
\int d^{2} \sigma A_{\mu}^{(b) M N}(b) \partial_{-} b^{\mu} \lambda_{+}^{M} \lambda_{+}^{N} .
$$

Including this coupling, the conditions for $(0,4)$ supersymmetry should be (more or less) that the sum of the bare gauge field $A^{(b)}$ and the ADHM gauge field is self-dual. Physically this is a necessary addition (e.g. in Minkowski space we could have other gluons propagating) but for purposes of constructing self-dual gauge fields $A^{(b)}$ can be set to zero. In terms of the $2 \mathrm{~d}$ field theory, in Euclidean four dimensions these couplings are redundant. 


\section{Type II}

Repeating the construction for a type II theory will produce $U(N)$ self-dual connections. The most direct analog of the type I construction is to take 8, 4 and 0-branes in type Ila theory. The world-volume theories are similar except that we do not impose the world-sheet orientation projection $\Omega=1$, and we reduce the dimension on each brane by one.

It is convenient to first derive the (unphysical) theory of 9,5 and 1 branes in type IIb and then do dimensional reduction. The basic 1-brane now has $(8,8)$ supersymmetry and there are right movers $\psi_{+}$and a $U(1)$ gauge field. The gauge fermions $\lambda$ are still chiral (this was due to GSO) but are now complex, as are the fields $\phi$ and $\chi$ and the couplings $h$. We distinguish fundamental and antifundamental indices $m M$ and $\bar{m} \bar{M}$. The full list of fields is

$$
\begin{array}{cccc}
\text { boson } & \text { fermion } & \text { sector } & \\
b^{A Y} & \psi_{-}^{A^{\prime} Y} & D D & \\
b^{A^{\prime} \tilde{A}^{\prime}} & \psi_{-}^{A} \tilde{A}^{\prime} & D D & \\
A_{--}, A_{++} & \psi_{+}^{A A^{\prime}}, \psi_{+}^{\tilde{A}^{\prime} Y} & D D & U(1) \text { gauge field and }(0,8) \text { gauginos } \\
\phi^{A^{\prime} \bar{m}} & \chi_{-}^{A \bar{m}} & D N 5 & 1-5 \text { strings (complex fields) } \\
\bar{\phi}^{A^{\prime} m} & \bar{\chi}_{-}^{A m} & D N 5 & \text { charge conjugates } \\
& \chi_{+}^{Y \bar{m}}, \bar{\chi}_{+}^{Y m} & D N 5 & \\
& \lambda_{+}^{\bar{M}}, \bar{\lambda}_{+}^{M} & D N 9 &
\end{array}
$$

The 15 sector now has $(4,4)$ world-sheet supersymmetry. The interactions include those in the gauged kinetic term, the $U(1)$ D-term and the gaugino couplings, and those following from the $(2,2)$-superspace superpotential $W=\left(X_{m n}^{A Y}-b^{A Y} \delta_{m n}\right) \phi_{A^{\prime} m} \phi^{A^{\prime} n}$ :

$$
\begin{aligned}
\mathcal{L}=\mathcal{L}_{k i n} & +\left|\phi_{1 m}\right|^{2}-\left|\phi_{2 m}\right|^{2}+\left(\psi_{-}^{A^{\prime} Y} \bar{\chi}_{Y+}^{m}+\psi_{+}^{A A^{\prime}} \bar{\chi}_{A-}^{m}\right) \phi_{A^{\prime} \bar{m}}+\text { c.c. } \\
& +\left(\phi_{A^{\prime} m} \bar{\phi}_{A^{\prime} \bar{m}}\right)^{2}+\phi_{A^{\prime} \bar{m}} \bar{\phi}^{A^{\prime} n}\left(X_{m \bar{n}}^{A Y}-b^{A Y} \delta_{m \bar{n}}\right)^{2}+ \\
& \chi_{-}^{A \bar{m}} \bar{\chi}_{+}^{Y n}\left(X_{m \bar{n}}^{A Y}-b^{A Y} \delta_{m \bar{n}}\right) .
\end{aligned}
$$

The extra $\phi^{4}$ potential terms have no effect on the branch $\phi=0$.

The 159 couplings are those following from a complex version of (2.8),

$$
\begin{aligned}
& C_{A A^{\prime}}^{M}=\bar{h}_{A m}^{\bar{M}} \phi_{A^{\prime} \bar{m}} \\
& \bar{C}_{A A^{\prime}}^{\bar{M}}=h_{A \bar{m}}^{M} \bar{\phi}_{A^{\prime} m}
\end{aligned}
$$

leading to the $\lambda$ Lagrangian

$$
\begin{aligned}
\mathcal{L}=\int d^{2} \sigma & \bar{\lambda}_{+}^{M}\left(\partial_{--}+i A_{--}\right) \lambda_{+}^{\bar{M}}+\lambda_{+}^{\bar{M}} \bar{\chi}_{m-}^{A} h_{A M}^{\bar{m}}+\text { c.c. } \\
& +\frac{1}{4} \int d^{2} \sigma \epsilon^{A B} \epsilon^{A^{\prime} B^{\prime}} C_{A A^{\prime}}^{M} C_{B B^{\prime}}^{\bar{M}}
\end{aligned}
$$


To verify $(0,4)$ supersymmetry, we follow the analysis in [8], promoting $\lambda$ and $C$ to complex fields. There is a new element, the world-sheet $U(1)$ gauge field $A$, but this can be accomodated as follows. We work with the $(0,4)$ supersymmetry transformation

$$
\begin{aligned}
\delta A_{++} & =i \eta_{+A A^{\prime}} \psi_{+}^{A A^{\prime}} \\
\delta A_{--} & =0 \\
\delta \psi_{+}^{A A^{\prime}} & =\eta_{+}^{A A^{\prime}} F
\end{aligned}
$$

with $F=\partial_{--} A_{++}-\partial_{++} A_{--}$. This commutes to a translation up to gauge transformation,

$$
\begin{aligned}
& {\left[\delta_{\eta^{\prime}}, \delta_{\eta}\right] A_{++}=-i \eta \eta^{\prime}\left(\partial_{--} A_{++}+\partial_{++} \epsilon\right)} \\
& {\left[\delta_{\eta^{\prime}}, \delta_{\eta}\right] A_{--}=-i \eta \eta^{\prime}\left(\partial_{--} A_{--}+\partial_{--} \epsilon\right)}
\end{aligned}
$$

with $\epsilon=-A_{--}$. This gauge transformation will also enter into the supersymmetry transformation for matter fermions; for example

$$
\delta \chi_{-}^{A \bar{m}}=\eta_{+}^{A A^{\prime}}\left(\partial_{--}+i A_{--}\right) \phi^{A^{\prime} \bar{m}} .
$$

The non-trivial verification is the on-shell $\lambda$ supersymmetry. Varying twice using (2.4) gives (as in [8] section 2.2)

$$
\begin{aligned}
{\left[\delta_{\eta^{\prime}}, \delta_{\eta}\right] \lambda_{+}^{\bar{M}} } & =-i \eta \eta^{\prime} G_{\theta}^{\bar{M}} \rho^{\theta} \\
& =-i \eta \eta^{\prime}\left(\partial_{--}+i A_{--}\right) \lambda_{+}^{\bar{M}}
\end{aligned}
$$

by the equations of motion. But this is the combined supersymmetry and gauge transformation (3.6).

We then derive the 0-brane theory by dimensional reduction. The only role of the $U(1)$ gauge field will be to restrict the allowed states of the 0-brane quantum mechanics to $U(1)$ neutral states such as the adjoint. It will not play a role in the construction of the self-dual connection, which proceeds in a way similar to the $S O(N)$ case.

The gauge fermions $\lambda$ have the following Yukawa couplings to $\chi$ :

$$
\left(\begin{array}{ll}
\bar{\chi}_{1} & \bar{\chi}_{2}
\end{array}\right)_{-} \cdot\left(\begin{array}{ccc}
X^{11}-b^{11} & X^{12}-b^{12} & h_{M}^{1 \bar{m}} \\
X^{21}-b^{21} & X^{22}-b^{22} & \left(h^{2}{ }^{t r}\right)_{\bar{m}}^{M}
\end{array}\right)\left(\begin{array}{c}
\chi^{1} \\
\chi^{2} \\
\lambda
\end{array}\right)_{+}
$$

defining an operator $\mathcal{D}^{+}$, such that the gauge bundle will be the kernel ker $\mathcal{D}^{+}$, spanned by the massless modes $\mathcal{D}^{+} v_{i}=0$. The complex conjugate fermions have similar Yukawa couplings involving the adjoint operator, $\bar{\lambda} \mathcal{D} \chi$, and in terms of $\mathcal{D}$ the gauge bundle will be the cokernel cok $\mathcal{D}$. The two definitions of subbundle agree, and the final Lagrangian can again be written in the form (2.12). 


\section{Conclusions}

In the present work, we explicitly verified the claim of Witten that instanton backgrounds in type I string theory are equivalent to configurations of Dirichlet 5-branes.

From the point of view of a type I fundamental string, an instanton background and a configuration of Dirichlet 5-branes act as two rather different-looking boundary conformal field theories; one with the action (2.13), the other with an action containing the DD and DN vertex operators for $X$ and $h$. Both must renormalize to the same boundary theory surely true on grounds of supersymmetry, but not easy to verify explicitly.

However, the effect of the D-brane configuration on the Dirichlet 1-brane is much simpler. Its world-sheet theory includes an "ADHM sigma model" sector, which reproduces the vector potential of the equivalent instanton background.

Combining this result with [7] provides an answer to a question raised by Witten in [8]: from the point of view of a string moving in an instanton background, the existence of a second branch of minima of the superpotential in the zero scale size limit, $b=0$ and $\phi \neq 0$, is rather mysterious. On this branch the string is equivalent to an instanton in the 5 -brane world-volume gauge theory. Of course, the metric on this moduli space is quantum corrected and it has been argued that the zero scale size limit is at infinite distance in the quantum moduli space [11].

The construction is clearly very general. For example, we believe it will be straightforward to apply it to the construction of $U(N)$ instanton moduli spaces on ALE spaces in [9], and reproduce Kronheimer and Nakajima's explicit construction of gauge fields and metrics 12,10.

In fact, the construction is more general than the ADHM construction, as it does not require hyperkähler structure on the moduli space, but only the ability to compute the superpotential of the resulting gauge theory. For example, placing 5-branes in various planes in six dimensions, say the 012345, 012367 and 012389 planes, will produce configurations equivalent to six-dimensional gauge fields preserving a supersymmetry, i.e. which solve the Donaldson-Uhlenbeck-Yau equation. The corresponding 1-brane sigma model will then provide a construction of the six-dimensional gauge connection. *

* Because this theory has only $(0,2)$ world-sheet supersymmetry, there might be world-sheet renormalization, and only in the IR limit are we guaranteed to get a solution of the DonaldsonUhlenbeck-Yau equation. It will be interesting to see if this is required, and if so, give a physical interpretation to this RG flow. We thank E. Witten for bringing up this point. 


\subsection{Dirichlet branes as local probes}

A more general lesson to draw from this work is that the Dirichlet brane can serve as a local probe, which can measure the fields at a point in space-time. Traditionally, a question such as "what is the metric (or gauge field) at the point $x$ " has been regarded as meaningless in string theory, because a fundamental string provides a probe which is necessarily smeared out over distances $\sqrt{\alpha^{\prime}}$. In calculations, this shows up in the fact that only on-shell vertex operators give uniquely defined amplitudes.

In string perturbation theory, a D-brane is effectively an infinitely massive object, whose center of mass motion depends on the fields (and perhaps finitely many derivatives) at a point. Thus its world-volume theory must - at least implicitly - encode this data.

Note that we are not claiming that the world-volume theory does not receive $\alpha^{\prime}$ corrections - rather, that the result of adding such corrections can be usefully described as a corrected local gauge field $A_{\mu}(x)$.

Can one define local fields by taking the limit $\lambda \rightarrow 0$ of finite coupling results? This seems plausible for gauge fields, but is not as obvious for the metric. For example, one might worry that an infinitely massive probe would necessarily affect the original background. This question deserves a detailed analysis (it is non-trivial in field theory as well).

We believe that the use of D-branes as probes will help clarify the geometric interpretation of other D-brane solutions as well.

We would like to thank G. Moore for some collaboration on this project, and M. Berkooz, R. Leigh, N. Nekrasov, S. Shatashvili and E. Witten for discussions. 


\section{References}

[1] J. Polchinski and E. Witten, "Evidence for Heterotic-Type I String Duality," hepth/9510169.

[2] J. Dai, R. G. Leigh and J. Polchinski, Mod. Phys. Lett. A4 (1989) 2073.

[3] J. Polchinski, Phys. Rev. Lett. 75 (1995) 4724-4727; hep-th/9510017.

[4] See S. Chaudhuri, C. Johnson, and J. Polchinski, "Notes on D-Branes," hepth/9602052, for a recent review.

[5] E. Witten, "Small Instantons in String Theory," hep-th/9511030.

[6] M. F. Atiyah, V. G. Drinfeld, N. J. Hitchin, and Y. I. Manin, Phys. Lett. A65 (1978) 185.

[7] M. R. Douglas, "Branes within Branes," hep-th/9512077.

[8] E. Witten, "Sigma Models and the ADHM Construction of Instantons," J. Geom. Phys. 15 (1995) 215-226.

[9] M. Douglas and G. Moore, "D-Branes, Quivers, and ALE Instantons," hep-th/9603167.

[10] M. Douglas, G. Moore, N. Nekrasov, and S. Shatashvili, "D-branes and the construction of $S O(w)$ instantons on ALE spaces," to appear

[11] E. Witten, "Some Comments On String Dynamics," hep-th/9507121.

[12] P.B. Kronheimer and H. Nakajima, "Yang-Mills instantons on ALE gravitational instantons," Math. Ann. 288 (1990) 263. 